International Journal of Engineering \& Technology, $9(2)(2020) 568-571$
International Journal of Engineering \& Technology
SPC
Website: www.sciencepubco.com/index.php/IJET
Research paper

\title{
Analysis of the time series of seismic and deformation monitoring, obtained from closed works at the Kirovsky mine of JSC "Apatite"
}

\author{
Gospodarikov Aleksandr P. ${ }^{1 *}$, Morozov Konstantin V. ${ }^{1}$, Revin Ilia E. ${ }^{1}$ \\ ${ }^{1}$ St. Petersburg mining University, Saint-Petersburg, Russia \\ *Corresponding author E-mail: kafmatem@spmi.ru
}

\begin{abstract}
The article is devoted to the analysis of the time series, obtained from seismic and deformation monitoring from closed works of Kukisvumchorr deposit JSC "Apatite". The objective of this study is to develop a method for assessing the results of monitoring geomechanical processes in the rock mass on the example of the Kirov mine JSC "Apatit". As a result of closed works, rock masses are changing its natural state of stress. This article has consistently outlined the use of machine learning algorithms in applied problems of geomechanics and geoinformatics. By comparing the schedule of mining operations and seismic activity data with time series of deformations, it is possible to obtain a functional relationship that predicts the distribution of deformations in the rock massif. The results of a computational experiment illustrating the possibility and feasibility of using machine learning algorithms in solving geomechanics problems are presented.
\end{abstract}

Keywords: Gradient Boosting Algorithm; Time Series; Deformation Monitoring; Khibiny Apatite-Nepheline Deposits.

\section{Introduction}

In the study of the stress state of the rock mass in its structure, it is possible to identify certain patterns [1]. Obtaining reliable predictive estimates of the values of the components of the stress, strain, and displacement vector tensors of a technologically disturbed rock mass is a highly relevant scientific and technical problem.

The task of predicting the basic parameters of the stress state of a rock massif, like any other problem arising in the process of working with large data arrays, depends both on the choice of the number and the type of approximating functions. Despite the significant number of formal quality metrics and methods for estimating parameters, for each specific data set, approximating models and criteria should be selected individually.

In our case, a time series (or a number of dynamics) is the statistical material collected at different points in time, the values of any parameters (in the simplest case of one) of the processes under study [9].Each unit of statistical material is called a measurement or reference; it is also possible to call it a level at a specified time. In the time series for each sample, the measurement time or the measurement number in order should be indicated. The time series differs significantly from simple data sampling, since such an analysis takes into account the interrelation of measurements with time, and not just the statistical diversity and statistical characteristics of the sample.

In other words, a time series is a sequence of values of a sensor ordered in time. Such data is easy to collect, and they are of high importance in determining the state of the system, since they can be obtained in real time. Thus, there is an acute problem of analyzing this type of data.

Moreover, the analysis of time series is a set of mathematical-statistical analysis methods designed to identify the structure of time series and to predict them.

These, in particular, include regression analysis methods. Identifying the structure of a time series is necessary to build a mathematical model of the phenomenon being studied - the source of the analyzed time series. This paper discusses various algorithms for determining anomalies in time series.

\section{Materials and methods}

An anomaly found in a time series can be determined in many ways. An event within a time series may be abnormal; a subsequence within a time series may be abnormal; or a whole time series may be abnormal relate to a set of normal time series.

For the detection of anomalous subsequences, the exact length of the sub-sequence is often unknown. Time series for training and testing can be of different lengths. The best similarity / difference measures that can be used for different types of time series are not easy to determine. Simple measures, such as the Euclidean distance, do not always work well, as they are very sensitive to outliers, and they are not valid when the time series have different lengths [2]. 
The accuracy of many anomaly detection algorithms is highly susceptible to noise in the data, since the separation of anomalies from noise is a complex task. Time series in real-world applications usually have a large size (length), and as the length increases, computational complexity increases. The accuracy of many anomaly detection algorithms is highly dependent on the correspondence of the time series scales, which is wrong for most data.

The anomaly search algorithms in this paper use data for training to study the model of normal behavior and to evaluate the anomalous nature of test data based on the model. Thus, the performance of any technique depends on the nature of the normal time series, as well as on the anomalous time series.

As an initial model, we consider the monitoring data of deformations from sensors No. 1-5 and monitornig data of seismic activity since November 2017 to July 2018.

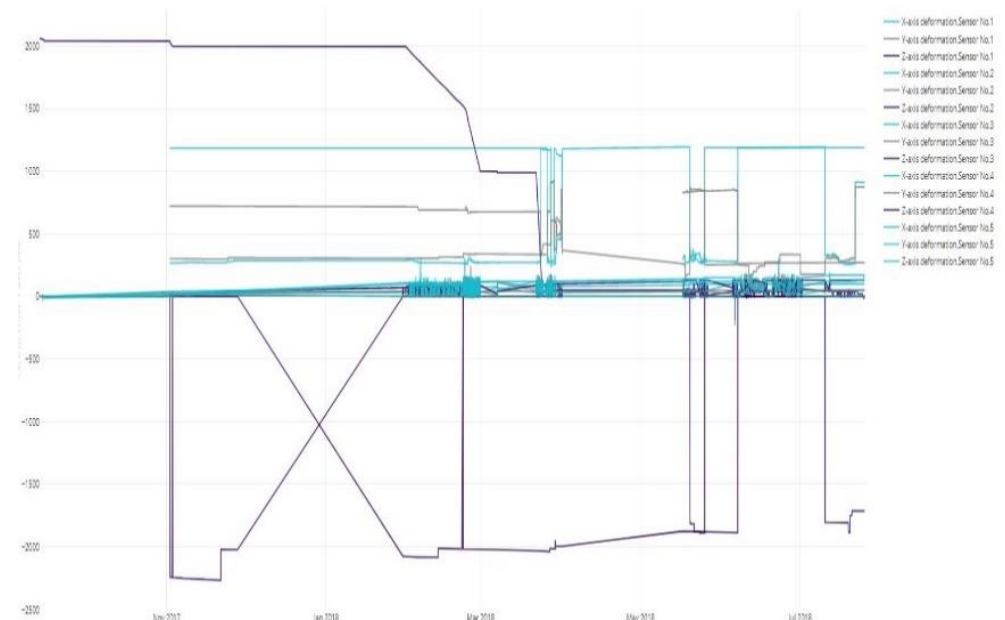

Fig. 1: Results of Deformation Monitoring Using Sensor No. 5, Taking into Account the Seismic Events Associated with the Maximum Increase in Deformations.

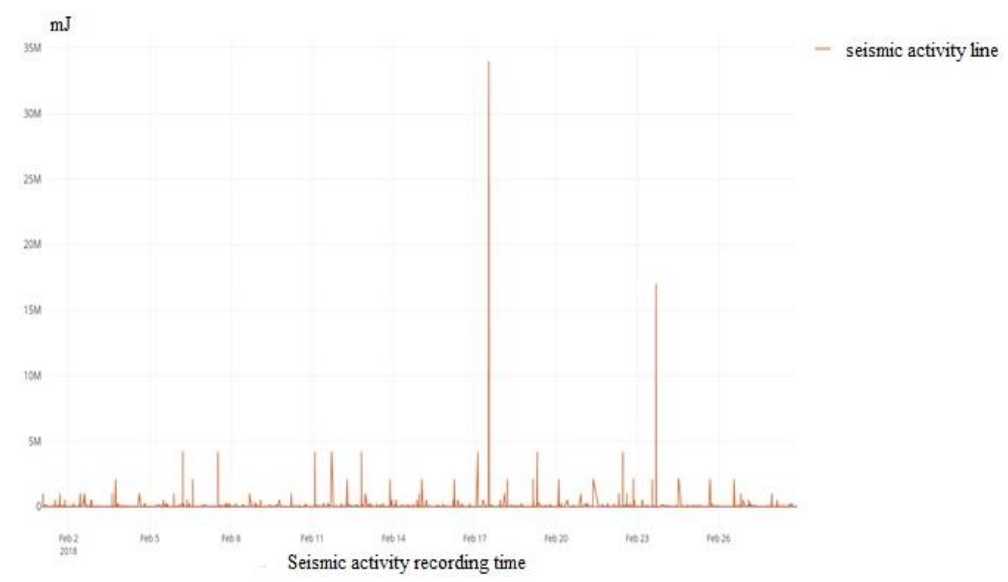

Fig. 2: Seismic Monitoring Data of the Kirov Mine JSC "Apatit" for February 2018.

As can be seen from the figure 1, the time series of deformations of these sensors have weak representative properties due to their nonstationarity. However, we can distinguish a certain subsequence, which will be considered further on the example of the deformation data obtained from sensor No. 5 .

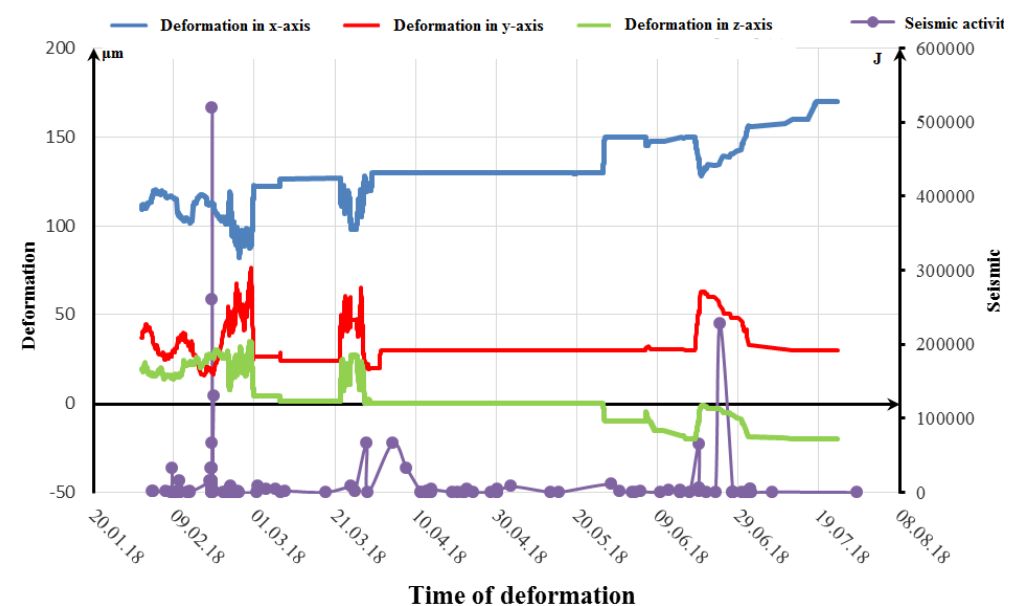

Fig. 3: Results of Deformation Monitoring Using Sensor No. 5, Taking into Account the Seismic Events Associated with the Maximum Increase in Deformations. 
Comparison of the data obtained as a result of modeling with the data of deformation monitoring allows us to draw the following conclusions:

1) In the rock mass, there is a connection between seismic events and deformation values. So, for example, deformation processes of a different nature originate at the same time for all wells: -22 February 07:01. At the same time, according to the seismic monitoring schedule for February 22, 2018, the largest event in terms of signature that day was recorded at 11:40 and was 4.2 MJ. The largest events in the reporting period are two seismic events: February 17 at 12: 23-34 MJ and February 23 at 16: 31-17 MJ. After the seismic event of February 23, there are no qualitative changes on the graphs of the strain values, while its signature is 4 times the event signature of February 22, which makes it possible to make several assumptions at once:

2) Since large seismic events have a rather long "response" in the array, the simultaneous onset of various deformation processes in sensors No. 1,2,3,5 from February 22 at 07:01 may be associated with a seismic event from February 17 at 12:23.

3) Based on the strain values obtained during laboratory tests, it can be concluded that all strain values exceeding $350 \mu \mathrm{m}$ are indications of the occurrence of inelastic deformations in the rock massif. Thus, the values recorded in 1 and 3 sensors, testifies to the possibility of the existence of such phenomena as the accumulation of potential energy that can be realized in the form of dynamic manifestations of rock pressure and the destruction of the array in the vicinity of the well.

\section{Results}

This section examines the problem of detecting anomalies for one-dimensional time series. There are many works on the search for anomalies [3], most of which use the approach of searching for objects that stand out from the general distribution. This strategy works well with the independence of objects in the training and test samples. The problem is that the time series has a time structure, and randomly mixing in the values of the entire series without saving this structure is impossible, otherwise the process will lose all the relationships of observations with each other.

The essence of the approach to the construction of the model - the training of the model begins on a small segment of the time series, from the beginning to some $t$, and then the forecast is made for $\mathrm{t}+\mathrm{n}$ steps forward and an error is considered. Further, the training sample is extended to $t+n$ values and is predicted from $t+n$ to $t+2 * n$ values. So the iteration of the test segment continues until the last available observation. As a result, we get as many samples as fit in the gap between the initial training segment and the entire length of the series.

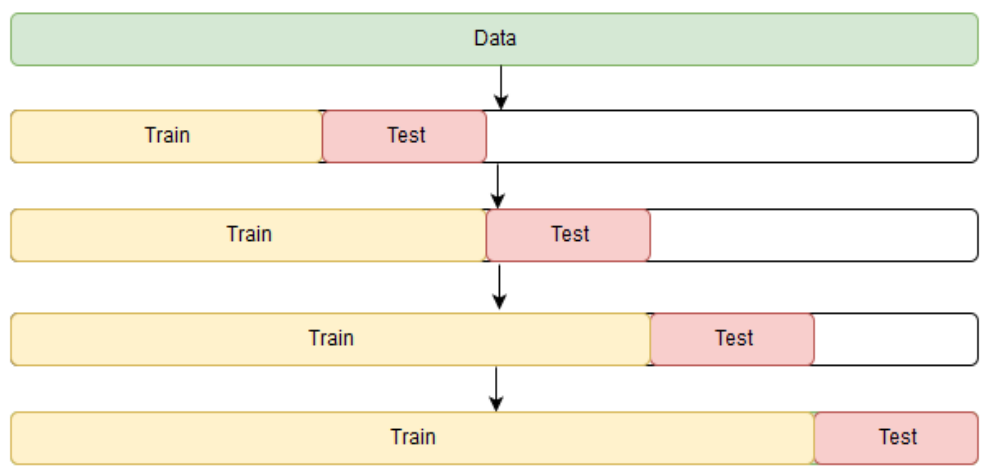

Fig. 4: Scheme for Teaching A Mathematical Model of Time Series Analysis.

Next, selection of the appropriate iterative algorithm used to solve the minimization problem. The gradient boosting algorithm is used as such an algorithm. First, it is necessary to calculate the gradient $\nabla \mathrm{L}_{\theta}(\hat{\theta})$ sum it with the iterative estimate along the gradient (with a minus sign).

Thus, the computational algorithm is organized as follows [4]:

- Initialize the initial approximation of the parameters $\hat{\theta}=\hat{\theta}_{0}$;

- For each iteration $\mathrm{t}=1, \ldots, \mathrm{M}$ calculate the gradient of the loss function $\nabla \mathrm{L}_{\theta}(\hat{\theta})$ at the current approximation of the parameter $\hat{\theta}$.

$\nabla \mathrm{L}_{\theta}(\widehat{\theta})=\left[\frac{\partial \mathrm{L}(\mathrm{y}, \mathrm{f}(\mathrm{x}, \theta)}{\partial \theta}\right]_{\theta=\widehat{\theta}}$

- Set the current iteration $\widehat{\theta_{t}}$ approximation based on the value of the calculated gradient

$\widehat{\theta_{\mathrm{t}}} \leftarrow \nabla \mathrm{L}_{\theta}(\hat{\theta})$

- Update parameter approximation $\hat{\theta}$, saving it

$\widehat{\theta}=\sum_{\mathrm{i}=0}^{\mathrm{M}} \hat{\theta}_{\mathrm{i}}$

Knowing the value of the loss function gradient, one can calculate its value for the given data. Learning the model is that our predictions are most correlated with the gradient value (with a minus sign). Both for classification, and for regression, and for ranking, the procedure of minimization of the square of the difference between pseudo-residues $r$ and the obtained predictions is applied 


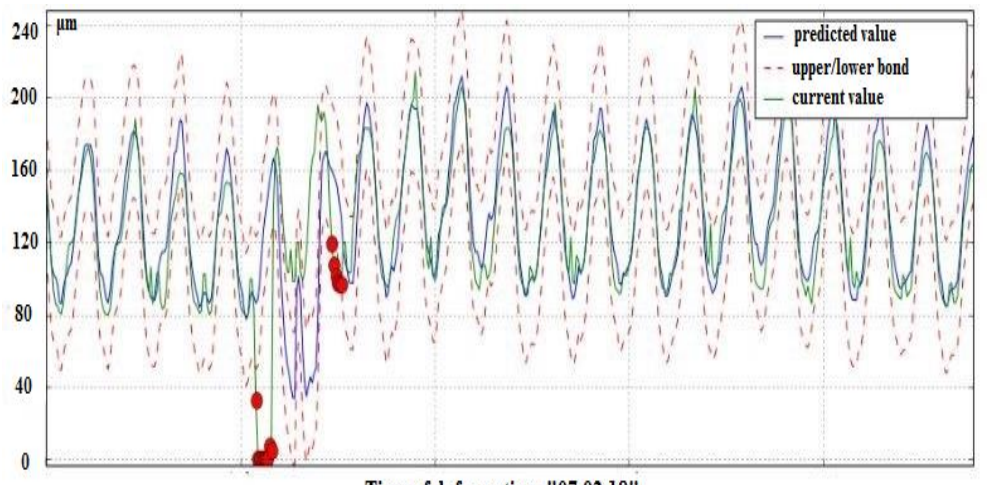

Time of deformation: "07.02.18"

Fig. 5: Analysis of the Time Series of Deformations Along the X-Axis Using the Gradient Boosting Method. 1 Day Was Taken as the Observation Period. Red Dots Indicate "Anomalous" Values.

As can be seen from figure 5, the model allows to determine confidence intervals (dotted line upper bond / lower bond). Then, at each step of the process iteration, the value of the loss function $\nabla \mathrm{L}_{\theta}(\widehat{\theta})$ is calculated. After that, the approximation of the parameters $\widehat{\theta} \leftarrow \widehat{\theta}+\widehat{\theta_{t}}=\sum_{i=0}^{\mathrm{M}} \widehat{\hat{\theta}_{i}}$ is updated. If the obtained value of the loss function is beyond the confidence interval, the point refers to the "anomalous" one [5]. With the help of the model, the points of release of the time series were refined, despite the fact that there was an error in predicting the series at the release point.

\section{Conclusions}

The time series analysis considered in this article have shown their suitability for the use of geomechanics and geomonitoring problems. Method have been proposed for constructing a mathematical model of a time series of deformation and its training on existing data. The one-dimensional time series analysis model considered in this article, based on the gradient-boosting algorithm, is an efficient algorithm for searching for "anomalies", allows smoothing time series, building confidence intervals and predictive models. The further development of time series analysis methods is the use of neural networks.

The choice of optimal neural network architecture is based on several factors. In the real world, we often deal with multiscale processes that have fast (long-term) and slow (short-term) subprocesses. Under these conditions, normal forward propagation neural networks usually show poor results. An accurate data-driven predictive model can be developed using a LSTM neural network. The proposed network architecture includes two LSTM layers with a linear output level. In addition, the prediction model uses a consistent LSTM network architecture. Long short-term memory (LSTM) is a special kind of recurrent neural network architecture capable of learning long-term dependencies. LSTMs are specifically designed to avoid the problem of long-term dependency (memorizing information for long periods of time).
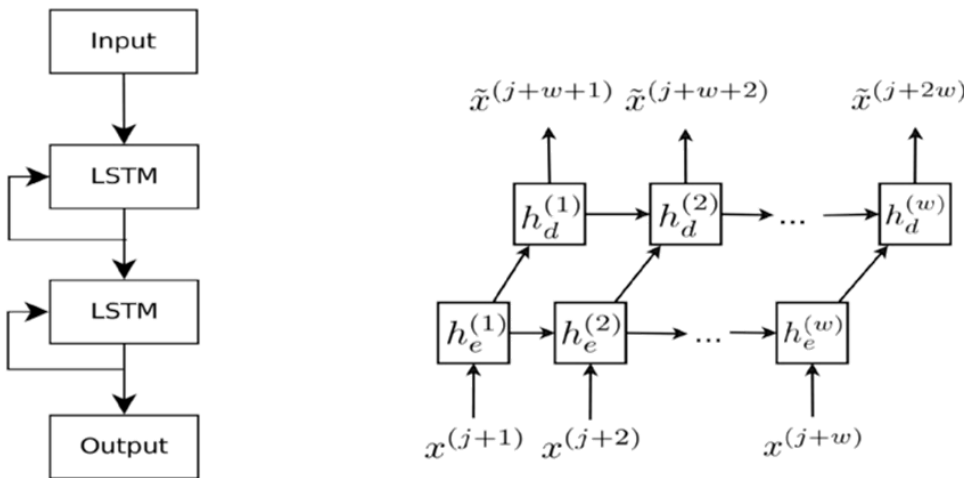

Fig. 6: Neural Network Architecture and Prediction Scheme Using LSTM Layers.

\section{References}

[1] Protosenya A. G. Study of compressive strength of fractured rock mass / A. G. Protosenya, P. E. Verbilo// Notes of the Mining Institute, 2017, Vol. 223- p. 51-57.

[2] Holt, Charles C. (January-March 2004). "Forecasting Trends and Seasonal by Exponentially Weighted Averages". International Journal of Forecasting. 20 (1): 5-10. https://doi.org/10.1016/j.ijforecast.2003.09.015.

[3] Brown, Robert Goodell (1963). Smoothing Forecasting and Prediction of Discrete Time Series. Englewood Cliffs, NJ: Prentice-Hall.

[4] Friedman J.H. Greedy Function Approximation: a Gradient Boosting Machine. Technical Report. Dept. of Statistics, Stanford University, 1999.

[5] Hastie, T.; Tibshirani, R.; Friedman, J. H. (2009). "10. Boosting and Additive Trees". The Elements of Statistical Learning (2nd ed.). New York: Springer. pp. 337-384. https://doi.org/10.1007/978-0-387-84858-7_10. 